\title{
MIMO nonlinear PID predictive controller
}

\author{
W. Feng, J. O'Reilly and D.J. Ballance
}

\begin{abstract}
A class of nonlinear generalised predictive controllers (NGPC) is derived for multiinput multi-output (MIMO) nonlinear systems with offset or steady-state response error. The MIMO composite controller consists of an optimal NGPC and a nonlinear disturbance observer. The design of the nonlinear disturbance observer to estimate the offset is particularly simple, as is the associated proof of overall nonlinear closed-loop system stability. Moreover, the transient error response of the disturbance observer can be arbitrarily specified by simple design parameters. Very satisfactory performance of the proposed MIMO nonlinear predictive controller is demonstrated for a three-link nonlinear robotic manipulator example.
\end{abstract}

\section{Introduction}

A class of nonlinear PID type controllers for nonlinear single-input single-output (SISO) systems with offset using a nonlinear generalised predictive control (NGPC) approach has recently been developed [1]. Offset or steady-state system response error in industrial control systems arises from many sources including load friction, intrinsic steady-state nonlinearity and uncertainties in system modelling $[1,2]$. Rather than introducing integral action directly to eliminate offset, the approach taken in [1] is to use a composite nonlinear controller, comprising an optimal NGPC and a nonlinear disturbance observer, whereby integral action arises naturally.

The great appeal of extending PID type control to nonlinear continuous-time systems by way of generalised predictive control such as [1] stems from three main reasons. First, PID control is widely used in industry because of its simple structure, ease of tuning, and its ability to remove offset $[3,4]$. Secondly, industrial processes are inherently continuous-time and nonlinear in their dynamical characteristics. Thirdly, generalised predictive control (GPC) is considered the industrial control method of choice in replacing PID control, especially in the process industries.

The nonlinear predictive control work $[1,2]$ can be thought of as an extension of earlier continuous-time work on NGPC [5-7] to handle system disturbances using a nonlinear observer. It can also be considered as a nonlinear continuous-time extension of the original discrete-time GPC proposed in the incremental control formulation by Clarke et al. [8] which has integral action and can remove offset. For more general background on GPC and it's extension to NGPC, the reader is referred to Chen et al. [1, 2].

Many nonlinear systems are inherently multi-input multi-output (MIMO) with significant dynamical cross-

(C) IEE, 2002

IEE Proceedings online no. 20020339

DOI: $10.1049 /$ ip-cta:20020339

Paper first received 26th June 2001 and in revised form Ist February 2002

The authors are with the Centre for Systems and Control, Faculty of Engineering, University of Glasgow, Glasgow G12 8LT, UK coupling between system inputs and outputs. A case in point is the independent joint control of a multilink robotic manipulator where there is significant dynamical crosscoupling between link angular displacements. Hence, the main purpose of the paper is to generalise the NGPC approach of [1] for SISO systems with offset to MIMO systems with offset.

An important part of the NGPC concerns the design of an appropriate nonlinear observer to estimate the disturbance affecting the MIMO system. This disturbance has different meanings in different applications [2]. For example, the system disturbance can represent friction in the independent joint control of a manipulator. A second purpose of the paper, therefore, is to significantly simplify the design of the nonlinear observer required to estimate the disturbance. As will be shown, this has the additional very important benefit of providing a simpler proof of overall nonlinear closed-loop system stability, even in the SISO case, than that considered in [1].

\section{Predictive controller}

The nonlinear dynamics of many mechanical systems can be described by a second-order matrix equation, given by

$$
\begin{aligned}
J(\theta(t), \dot{\theta}(t)) \ddot{\theta}(t) & +G(\theta(t), \dot{\theta}(t)) \\
& =B_{1}(\theta(t), \dot{\theta}(t)) u(t)+B_{2}(\theta(t), \dot{\theta}(t)) d(t)
\end{aligned}
$$

where $\theta \in R^{n}$ denotes the angular displacements of the mechanical systems and is observable. $n$ is the number of degrees of freedom of the mechanical system, that is, the number of links in the multilink manipulator. $u(t) \in R^{m}$ denotes the vector of generalised torques and forces. $d \in R^{n}$ represents the vector of disturbance affecting the system (1). $J \in R^{n \times n}$ denotes the inertia matrix and is invertible. $G \in R^{n}$ consists of Coriolis, centrifugal and gravitational terms etc. $B_{1} \in R^{n \times m}$ denotes the input torque gain matrix and is invertible. $B_{2} \in R^{n \times n}$ denotes the disturbance gain matrix and is invertible. The controlled output is a linear combination of the displacements of the mechanical system, i.e.

$$
y=D \theta(t)
$$

where $y \in R^{n}$. 
For simplicity, only the case $m=n$ is discussed in the following but all results developed in this paper can be extended to the case $m<n$ where the zero dynamics of the nonlinear system (1) are stable. Hence, without loss of generality, it is assumed $D=I$, that is

$$
y=\theta(t)
$$

Consider the nonlinear system (1) with the following performance index:

$$
J=\frac{1}{2} \int_{T_{1}}^{T_{2}}\left(y(t+\tau)-\theta^{d}(t+\tau)\right)^{T}\left(y(t+\tau)-\theta^{d}(t+\tau)\right) d \tau
$$

where $\theta^{d}$ is the vector of desired or command angular displacements and $T_{1}$ and $T_{2}$ are the minimum and maximum predictive control times, respectively. Following Gawthrop et al. [7] a control weighting term is not included in the performance index (4); rather, a weighting can be achieved by $T_{1}$ and $T_{2}$ in GPC. Another way to limit the control action effect is to use $P$ polynomials [7].

Differentiating the output $y(t)$ of (3) twice with respect to time, together with substitution of the system (1) gives

$$
\begin{gathered}
\dot{y}(t)=\dot{\theta}(t) \\
\ddot{y}(t)=\ddot{\theta}=J(\theta, \dot{\theta})^{-1}\left(-G(\theta, \dot{\theta})+B_{1}(\theta, \dot{\theta}) u(t)\right. \\
\left.+B_{2}(\theta, \dot{\theta}) d(t)\right)
\end{gathered}
$$

Combining (6) with the output equation (3) yields

$$
\begin{aligned}
\bar{Y}=\left[\begin{array}{c}
y(t) \\
\dot{y}(t) \\
\ddot{y}(t)
\end{array}\right]= & M(\theta, \dot{\theta})+\left[\begin{array}{c}
0 \\
0 \\
N_{1}(\theta, \dot{\theta})
\end{array}\right] u(t) \\
& +\left[\begin{array}{c}
0 \\
0 \\
N_{2}(\theta, \dot{\theta})
\end{array}\right] d(t)
\end{aligned}
$$

where

$$
\begin{aligned}
& N_{1}(\theta, \dot{\theta})=J(\theta, \dot{\theta})^{-1} B_{1}(\theta, \dot{\theta}) \\
& N_{2}(\theta, \dot{\theta})=J(\theta, \dot{\theta})^{-1} B_{2}(\theta, \dot{\theta}) \\
& M=\left[\begin{array}{c}
0 \\
\dot{\theta} \\
-J(\theta, \dot{\theta})^{-1} G(\theta, \dot{\theta})
\end{array}\right]
\end{aligned}
$$

A Taylor expansion of the output $y(t+\tau)$ and command signal $\theta^{d}(t+\tau)$ at time $t$ up to second order yields

$$
\begin{aligned}
y(t+\tau) & =H(\tau) \bar{Y}(t) \\
\theta^{d}(t+\tau) & =H(\tau) \bar{W}(t)
\end{aligned}
$$

where

$$
\bar{W}(t)=\left[\begin{array}{c}
\theta^{d}(t) \\
\dot{\theta}^{d}(t) \\
\ddot{\theta}^{d}(t)
\end{array}\right]
$$

The $n \times 3 n$ matrix $H(\tau)$ in (11) and (12) is given by

$$
H(\tau)=\left[\begin{array}{lll}
I & \bar{\tau} & \bar{\tau}^{2} / 2 !
\end{array}\right]
$$

where the $n \times n$ matrix $\bar{\tau}$ is defined by

$$
\bar{\tau}=\operatorname{diag}\{\tau, \ldots, \tau\}
$$

Hence, after expanding the performance index (4) up to second order, it can be written as

$$
J=\frac{1}{2} \int_{T_{1}}^{T_{2}}(\bar{Y}(t)-\bar{W}(t))^{T} H(\tau)^{T} H(\tau)(\bar{Y}(t)-\bar{W}(t)) d \tau
$$

which upon integration yields

$$
J=\frac{1}{2}(\bar{Y}(t)-\bar{W}(t))^{T} \bar{H}\left(T_{1}, T_{2}\right)(\bar{Y}(t)-\bar{W}(t))
$$

where

$$
\bar{H}\left(T_{1}, T_{2}\right)=\int_{T_{1}}^{T_{2}} H(\tau)^{T} H(\tau) d \tau
$$

It follows from (14), (15) and (17) that

$$
\bar{H}\left(T_{1}, T_{2}\right)=\left[\begin{array}{lll}
\bar{H}_{11} & \bar{H}_{12} & \bar{H}_{13} \\
\bar{H}_{21} & \bar{H}_{22} & \bar{H}_{23} \\
\bar{H}_{31} & \bar{H}_{32} & \bar{H}_{33}
\end{array}\right]
$$

where

$$
\vec{H}_{i j}\left(T_{1}, T_{2}\right)=\frac{\bar{H}_{2}^{i+j-1}-\bar{H}_{1}^{i+j-1}}{(i-1) !(j-1) !(i+j-1)}
$$

and the diagonal matrices $\bar{H}_{1}$ and $\bar{H}_{2}$ in (20) are obtained by replacing $\tau$ with $T_{1}$ and $T_{2}$ in (15), respectively. Substitution of (7) into the cost (16) and minimisation of the cost (16) with respect to the control, $u(t)$, gives

$$
\begin{aligned}
u= & -\left(\left[\begin{array}{l}
0 \\
0 \\
N_{1}
\end{array}\right]^{T} \bar{H}\left(T_{1}, T_{2}\right)\left[\begin{array}{l}
0 \\
0 \\
N_{1}
\end{array}\right]\right)^{-1}\left[\begin{array}{l}
0 \\
0 \\
N_{1}
\end{array}\right]^{T} \\
& \times \bar{H}\left(T_{1}, T_{2}\right)\left(M+\left[\begin{array}{l}
0 \\
0 \\
N_{2}
\end{array}\right] d-\bar{W}\right)
\end{aligned}
$$

It can be shown that the matrix $\bar{H}_{33}$ is positive definite and hence invertible. Also noting it is assumed that the control $u(t)$ can be fully recovered from (1), this implies that $B_{1}(\theta, \dot{\theta})$, for all allowable $\theta$ and $\dot{\theta}$, is invertible. Using (8)-(10), the optimal control (21) can be written as

$$
\begin{aligned}
u= & -B_{1}(\theta, \dot{\theta})^{-1} J(\theta, \dot{\theta}) \vec{H}_{33}^{-1}\left[\begin{array}{lll}
\bar{H}_{31} & \bar{H}_{32} & \bar{H}_{33}
\end{array}\right] \\
& \times\left[\begin{array}{c}
\theta-\theta^{d} \\
\dot{\theta}-\dot{\theta}^{d} \\
J(0, \dot{\theta})^{-1}\left(-G(\theta, \dot{\theta})+B_{2}(\theta, \dot{\theta}) d\right)-\ddot{\theta}^{d}
\end{array}\right]
\end{aligned}
$$

Let

$$
\begin{aligned}
& K_{\mathrm{I}}=\bar{H}_{33}^{-1} \bar{H}_{31} \\
& K_{2}=\bar{H}_{33}^{-1} \bar{H}_{32}
\end{aligned}
$$

The control (22) further becomes

$$
\begin{aligned}
u= & -B_{1}(\theta, \dot{\theta})^{-1} J(\theta, \dot{\theta})\left[K_{1}\left(\theta-\theta^{d}\right)+K_{2}\left(\dot{\theta}-\dot{\theta}^{d}\right)\right. \\
& \left.+J(\theta, \dot{\theta})^{-1}\left(-G(\theta, \dot{\theta})+B_{2}(\theta, \dot{\theta}) d\right)-\ddot{\theta}^{d}\right] \\
= & B_{1}(\theta, \dot{\theta})^{-1}\left\{J(\theta, \dot{\theta})\left[K_{1}\left(\theta^{d}-\theta\right)+K_{2}\left(\dot{\theta}^{d}-\dot{\theta}\right)+\ddot{\theta}^{d}\right]\right. \\
& \left.+G(\theta, \dot{\theta})-B_{2}(\theta, \dot{\theta}) d\right\}
\end{aligned}
$$

It is observed in (23), (24) and (18)-(20), that the gain matrices $K_{\mathrm{t}}$ and $K_{2}$ explicitly depend on the choice of the predictive times $T_{1}$ and $T_{2}$. By adjusting these two design parameters $T_{1}$ and $T_{2}$, the desired system response can be achieved. In the next section, a nonlinear PID controller 
will be derived incorporating a nonlinear disturbance observer.

\section{Nonlinear disturbance observer}

In general, the design of an observer for nonlinear systems is a nontrivial exercise [9]. In this paper, since all states in (1) are observable and disturbances $d$ are supposed to be observed, a basic idea in the design of observers/ estimators is to characterise the estimation process by the difference between the estimated output and the actual output. Since (1) can be written as

$$
\begin{aligned}
d= & B_{2}(\theta(t), \dot{\theta}(t))^{-1}[J(\theta(t), \dot{\theta}(t)) \ddot{\theta}(t) \\
& \left.+G(\theta(t), \dot{\theta}(t))-B_{1}(\theta(t), \dot{\theta}(t)) u(t)\right]
\end{aligned}
$$

a nonlinear disturbance observer is proposed as

$$
\begin{aligned}
\dot{\bar{d}}= & -L(\theta(t), \dot{\theta}(t)) \hat{d}+L(\theta(t), \dot{\theta}(t)) B_{2}(\theta(t), \dot{\theta}(t))^{-1} \\
& \times\left[J(\theta(t), \dot{\theta}(t)) \ddot{\theta}(t)+G(\theta(t), \dot{\theta}(t))-B_{1}(\theta(t), \dot{\theta}(t)) u(t)\right]
\end{aligned}
$$

Since, in general, there is no prior information about the derivative of the disturbance $d$, it is reasonable to suppose that

$$
\dot{d}=0
$$

which implies that the disturbance varies slowly relative to the observer dynamics. However, it will be shown in Section 6 that the observer developed can track some fast time-varying disturbances when parameters of the observer are properly chosen.

The observer error is defined as

$$
\varepsilon(t)=d-\hat{d}
$$

By (26)-(29), we have

$$
\dot{\varepsilon}(t)=\dot{d}-\dot{\hat{d}}=L(\theta, \dot{\theta}) \hat{d}-L(\theta, \dot{\theta}) d
$$

Hence, by (29) and (30), the error dynamics of the observer are given by

$$
\dot{\varepsilon}+L(\theta, \dot{\theta}) \varepsilon=0
$$

It follows therefore from (31) that the nonlinear disturbance observer given in (27) is globally asymptotically stable, for all allowable $\theta$ and $\dot{\theta}$, if and only if $-L(\theta, \dot{\theta})$ is a Hurwitz matrix; that is, if and only if the real parts of all eigenvalues (real or complex) of $-L(\theta, \dot{0})$ are strictly negative. In meeting this stability requirement, the matrix $L(\theta, \dot{\theta})$ can assume many different forms. A particularly simple choice of $L(\theta, \dot{\theta})$ for stability is

$$
L(\theta, \dot{\theta})=C=\operatorname{diag}\left\{c_{1}, \ldots, c_{i}, \ldots, c_{n}\right\}
$$

where $c_{i}>0$, and the exponential convergence rate of the observer error dynamics (31) can be specified by appropriate choice of $c_{i}$. This implies that different convergence rates can be specified for different disturbances in the disturbance vector $d \in R^{n}$ in (1).

The acceleration signal $\ddot{\theta}$ is not available in many mechanical systems, and it is also impractical to construct the acceleration signal from the velocity signal by differentiation due to measurement noise. Although the observer (27) is not practical to implement, it provides a basis for further nonlinear observer design. Chen et al. $[1,2]$ have suggested a modified nonlinear disturbance observer as

$$
\begin{gathered}
\dot{z}=-L(\theta, \dot{0}) z+L(\theta, \dot{\theta})\left(G(\theta, \dot{\theta})-B_{1} u-p(\theta, \dot{\theta})\right) \\
\hat{d}=-B_{2}^{-1}(z+p(\theta, \dot{\theta}))
\end{gathered}
$$

As pointed out by Chen et al. [1,2], the observer (33), (34) is specified by the proper choice of the design function vector $p(\theta, \dot{\theta})$. It is a nontrivial job to find a $p(\theta, \dot{\theta})$ which can lead to a stable observer in the case of the MIMO problem at hand.

A better alternate strategy is that the nonlinear controller be realised by substituting the control signal (25) directly into the initial observer (27), where $d$ in (25) is replaced by its estimate $\hat{d}$. It is calculated as follows:

$$
\begin{aligned}
\dot{\bar{d}}= & -L(\theta(t), \dot{\theta}(t)) \hat{d}+L(0(t), \dot{\theta}(t)) B_{2}(\theta(t), \dot{\theta}(t))^{-1} \\
& \times\{J(\theta(t), \dot{\theta}(t)) \ddot{\theta}(t) \\
& +G(\theta(t), \dot{\theta}(t))-B_{1}(\theta(t), \dot{\theta}(t)) B_{1}(\theta(t), \dot{\theta}(t))^{-1} \\
& \times\left[J ( \theta ( t ) , \dot { \theta } ( t ) ) \left(K_{1}\left(\theta-\theta^{d}\right)\right.\right. \\
& \left.\left.\left.+K_{2}\left(\dot{\theta}-\dot{\theta}^{d}\right)+\ddot{\theta}^{d}\right)+G(\theta(t), \dot{\theta}(t))-B_{2}(\theta(t), \dot{\theta}(t)) \hat{d}\right]\right\} \\
= & -L(\theta(t), \dot{\theta}(t)) B_{2}(\theta(t), \dot{\theta}(t))^{-1}\left[J ( \theta ( t ) , \dot { \theta } ( t ) ) \left(K_{1}\left(0-\theta^{d}\right)\right.\right. \\
& \left.\left.+K_{2}\left(\dot{\theta}-\dot{\theta}^{d}\right)+\ddot{\theta}-\ddot{\theta}^{d}\right)\right]
\end{aligned}
$$

By integration of (35)

$$
\begin{aligned}
\hat{d}= & -L(\theta(t), \dot{\theta}(t)) B_{2}(\theta(t), \dot{\theta}(t))^{-1} \\
& \times\left[J(\theta(t), \dot{\theta}(t))\left(K_{1} \int_{0}^{t} e d \tau+K_{2} \int_{0}^{t} \dot{e} d \tau+\int_{0}^{t} \ddot{e} d \tau\right)\right] \\
& +\hat{d}(0)
\end{aligned}
$$

where we define the tracking error as

$$
e(t)=\theta-\theta^{d}
$$

Supposing the command signal $\theta^{d}$ is differentiable up to second order and if $\hat{d}(0)$ is chosen as

$$
\begin{aligned}
\hat{d}(0)= & -L(\theta(t), \dot{\theta}(t)) B_{2}(\theta(t), \dot{\theta}(t))^{-1} \\
& \times[J(\theta(t), \dot{\theta}(t))(e(0)+\dot{e}(0))]
\end{aligned}
$$

the disturbance estimate $\hat{d}(t)$ is given by

$$
\begin{aligned}
\hat{d}= & -L(\theta(t), \dot{\theta}(t)) B_{2}(\theta(t), \dot{\theta}(t))^{-1} \\
& \times\left[J(0(t), \dot{\theta}(t))\left(\dot{e}(t)+K_{2} e(t)+K_{1} \int_{0}^{t} e(\tau) d \tau\right)\right]
\end{aligned}
$$

\section{Nonlinear PID predictive controller}

If the disturbance estimate $\hat{d}$ of (39) is substituted for $d$ in (25), then a nonlinear PID predictive controller can be achieved as

$$
u=P(\theta, \dot{\theta}) e(t)+D(\theta, \dot{\theta}) \dot{e}(t)+I(\theta, \dot{\theta}) \int_{0}^{t} e(\tau) d \tau+N(\theta, \dot{\theta})
$$

where

$$
\begin{aligned}
P(\theta, \dot{\theta})= & B_{1}(\theta, \dot{\theta})^{-1}\left[J(\theta, \dot{\theta}) K_{1}\right. \\
+ & \left.B_{2}(\theta, \dot{\theta}) L(\theta, \dot{\theta}) B_{2}(\theta, \dot{\theta})^{-1} J(\theta, \dot{\theta}) K_{2}\right] \\
D(\theta, \dot{\theta})= & B_{1}(0, \dot{\theta})^{-1}\left[J(\theta, \dot{\theta}) K_{2}\right. \\
& \left.+B_{2}(\theta, \dot{\theta}) L(\theta, \dot{\theta}) B_{2}(\theta, \dot{\theta})^{-1} J(\theta, \dot{\theta})\right]
\end{aligned}
$$

$I(\theta, \dot{\theta})=B_{1}(\theta, \dot{\theta})^{-1} B_{2}(\theta, \dot{\theta}) L(\theta, \dot{\theta}) B_{2}(\theta, \dot{0})^{-1} J(\theta, \dot{\theta}) K_{1}$

$$
N(\theta, \dot{\theta})=B_{1}(\theta, \dot{\theta})^{-1}\left[J(0, \dot{\theta}) \ddot{\theta}^{d}+G(\theta, \dot{\theta})\right]
$$


This controller (40) can be thought of as a nonlinear PID predictive controller involving the state $x$ where $x=[\theta, \dot{\theta}]$ denotes the state vector of the mechanical system. The proportional and differential terms in (40) are nonlinear functions of the angular displacements of the mechanical system. In addition to traditional PID structure, a prediction part $N(x)$ given by (44) is included in the controller (40). The first term $B_{1}(\theta, \dot{\theta})^{-1} J(\theta, \dot{\theta}) \ddot{\theta}^{d}$ of (44) takes into account the control input requirement for future outputs using a second-order derivative of the reference signal. The second term $B_{1}(\theta, \dot{\theta})^{-1} G(\theta, \dot{\theta})$ of (44) is to compensate for the influence of the current system's dynamics on the future output and the input requirement for tracking future reference signals.

\section{Dynamics of the closed-loop system under MIMO predictive control}

Ensuring stability of the closed-loop system is one of the main problems in GPC. In this Section, it is shown that the closed-loop system under controller (40) incorporating a disturbance observer is asymptotically stable. For simplicity, we assume $T_{1}=0$ and $T_{2}=T$, bearing in mind that all the results below can be extended to the case $T_{1} \neq 0$.

The closed-loop system of the plant (1) and the controller (40) can be written as

$$
J(\theta, \dot{\theta})\left(\ddot{e}+\left(K_{2}+C\right) \dot{e}+\left(K_{1}+C K_{2}\right) e+C K_{1} \int_{0}^{t} e(\tau) d \tau\right)
$$

Combining (23), (24) and (20) yields

$$
\begin{gathered}
K_{1}=10 / 3 \bar{H}_{2}^{-2} \\
K_{2}=5 / 2 \bar{H}_{2}^{-1}
\end{gathered}
$$

Since $\bar{H}_{2}$ is a diagonal matrix, there are

$$
\begin{aligned}
K_{1}(i, i)=\frac{10}{3 T^{2}}, \quad i=1, \ldots, n ; \\
K_{1}(i, j)=0, \quad i, j=1, \ldots, n ; \quad i \neq j \\
K_{2}(i, i)=\frac{5}{2 T}, \quad i=1, \ldots, n ; \\
K_{2}(i, j)=0, \quad i, j=1, \ldots, n ; \quad i \neq j
\end{aligned}
$$

Thus the closed-loop error equation (45) decouples into the following equations

$$
\begin{aligned}
\ddot{e}_{i}+\left(\frac{2.5}{T}+c_{i}\right) & \dot{e}_{i}+\left(\frac{10}{3 T^{2}}+c_{i} \frac{2.5}{T}\right) e_{i} \\
& +c_{i} \frac{10}{3 T^{2}} \int_{0}^{t} e_{i}(\tau) d \tau=0, \quad i=1, \ldots, n
\end{aligned}
$$

Associated with (50), we have the closed-loop system characteristic equations

$$
\begin{array}{r}
s^{3}+\left(\frac{2.5}{T}+c_{i}\right) s^{2}+\left(\frac{10}{3 T^{2}}+c_{i} \frac{2.5}{T}\right) s+c_{i} \frac{10}{3 T^{2}}=0, \\
i=1, \ldots, n
\end{array}
$$

with characteristic roots (poles)

$s_{1}=-c_{i}, \quad s_{2,3}=\frac{-1.25 \pm j 1.3307}{T}, \quad i=1, \ldots, n$

Examining the poles of the closed-loop system in (52), it can be seen that:
(1) the closed-loop system is stable, since $c_{i}$ and $T$ are each positive

(2) if $c_{i}$ is chosen relatively large compared with the other two poles, it has relatively little overall impact on the system response and the damping ratio of the closed system is fixed at 0.684

(3) with a shorter predictive time $T$, the tracking error of (50) or equivalently (45) converges to zero faster.

\section{Illustrative MIMO nonlinear manipulator example}

In this Section, the nonlinear PID predictive control methods developed for second-order MIMO systems are applied to the independent joint control of a three joint revolute robot manipulator in a horizontal plane. A DC motor directly drives each link independently. Potentiometers and tachometers can measure the displacement and the velocity of each link, so that all the states of the robot are observable.

The MIMO nonlinear dynamics of the robot have been described by Lynch et al. [10]

$$
M(\theta) \ddot{\theta}+\dot{\theta}^{T} \Gamma(\theta) \dot{\theta}=\tau+d
$$

where the inertia matrix $M(\theta)$ and $\Gamma(\theta)$ are as defined in [10], for which the physical parameters are as listed in Table 1. Also in (53), the vector $\tau$ represents the input torques. The vector $d$ represents the friction, which includes Coulomb and viscous friction given by

$$
d(\dot{\theta})=z \operatorname{sign}(\dot{\theta})+k \dot{\theta}
$$

for which the parameters for the first, second and third links are given in Table 1.

The more comprehensive revised friction model [2] is used

$$
d_{a}=d+\left(T_{a}-d\right) e^{-(\dot{\theta} / l)^{2}}
$$

where $d \in R^{3}$ is the friction vector, $d_{a} \in R^{3}$ is the revised friction vector, and $l$ is a small positive scalar taken as 0.01 .

Comparing the robot model (53) with the general MIMO second-order model (1), the general nonlinear predictive PID controller given in (40) can be used for (53) by substituting $M(\theta)$ for $J(\theta, \dot{\theta}), \dot{\theta}^{T} \Gamma(\theta) \dot{\theta}$ for $G(\theta, \dot{\theta})$, the unit matrix $I$ for $B_{1}(\theta, \dot{\theta})$ and $B_{2}(\theta, \dot{\theta})$, and the torque inputs $\tau$ for control $u$.

Suppose that the minimum and maximum predictive control times in the performance index (4) are specified as $T_{1}=0$ and $T_{2}=1$. From (48) and (49), we have $K_{1}=\operatorname{diag}\{10 / 3,10 / 3,10 / 3\}$, and $K_{2}=\operatorname{diag}\{2.5,2.5$, $2.5\}$, and $L(\theta, \theta)$ in (32) is specified as $\operatorname{diag}\{25,25,30\}$.

Table 1: Kinematic parameters for simulated three-link robot. $m_{i}$ is the mass of link $i, l_{i}$ is the length of link $i, r_{i}$ is the distance from joint $i$ to the centre of link $i_{r}$ and $l_{i}$ is the inertia of link $i$ about its centre of mass. $z$ and $k$ are as defined in (54)

\begin{tabular}{lllllll}
\hline $\operatorname{Link}(i)$ & $l_{i}$ & $r_{i}$ & $m_{i}$ & $l_{i}$ & $z$ & $k$ \\
\hline 1 & 0.6 & 0.3 & 4.0 & 0.8 & 0.141 & 0.176 \\
2 & 0.3 & 0.15 & 2.0 & 0.5 & 0.0576 & 0.052 \\
3 & - & 0.15 & 0.5 & 0.125 & 0.015 & 0.01 \\
\hline
\end{tabular}

IEE Proc.-Control Theory Appl., Vol. 149, No. 3, May 2002 
To compare this nonlinear PID controller with the well known computed torque controller [11] given by

$$
\tau=M(\theta)\left(K_{1} e(t)+K_{2} \dot{e}(t)+\ddot{\theta}_{d}\right)+\dot{\theta}^{T} T(\theta, \dot{\theta}) \dot{\theta}
$$

where $K_{1}$ and $K_{2}$ are defined above, simulations are performed for both controllers. Command signals are three unit steps applied to link 1, link 2 and link 3 separately at $t=0,10$ and 20, respectively. Angular displacement responses are given in Figs. 1-3.

From Figs. 1-3, it is observed that the nonlinear PID controller has better performance than the classical computed torque method with particular regard to transient step response, good disturbance rejection, low interaction between individual links and zero steady-state error. Since the minimum and maximum prediction time in performance index (4) are chosen as $T_{1}=0$ and $T_{2}=1$, following the discussion in Section 5, the closed system should have the pair of poles $-1.25 \pm j 1.3307$. This is confirmed in Figs. 1-3. To show the effect of a change of predictive time to $T_{1}=0$ and $T_{2}=1.5$, minimum and maximum predictive time, respectively, from (55) and (56), we have $K_{1}=$ $\operatorname{diag}\left\{10 /\left(3 \times 1.5^{2}\right), \quad 10 /\left(3 \times 1.5^{2}\right), \quad 10 /\left(3 \times 1.5^{2}\right)\right\}$ and $K_{2}=\operatorname{diag}\{5 / 3,5 / 3,5 / 3\}$. Correspondingly, $L(\theta, \dot{\theta})$ in (32) is specified as $\operatorname{diag}\left\{25 \times 1.5^{2}, 25 \times 1.5^{2}, 30 \times 1.5^{2}\right\}$, which means that the integral action term $I(\theta, \dot{\theta})$ of $(40)$ is taken to be the same, as with the previous prediction times $T_{1}=0$ and $T_{2}=1$. Figs. $4-6$ corroborate the discussion in Section 5, that the closed-loop system has the pair of poles

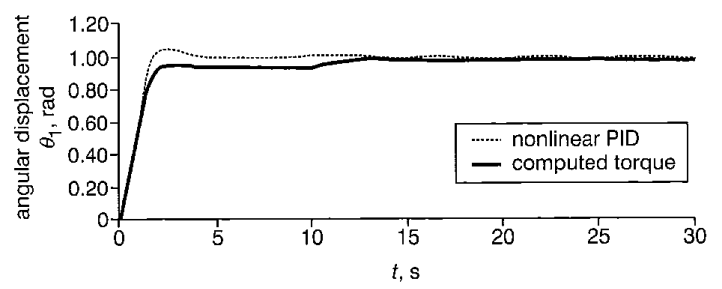

Fig. 1 Link 1, angular displacement step response for predictive times $T_{1}=0, T_{2}=1$

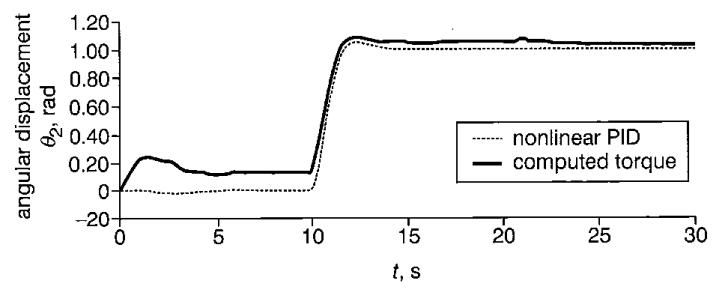

Fig. 2 Link 2, angular displacement step response for predictive times $T_{1}=0, T_{2}=1$

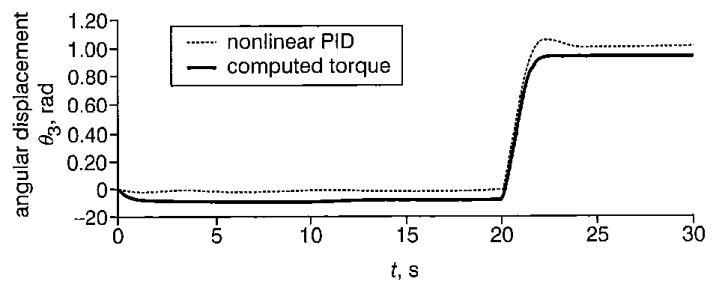

Fig. 3 Link 3, angular displacement step response for predictive times $T_{1}=0, T_{2}=1$

IEE Proc.-Control Theory Appl., Vol. 149, No. 3, May 2002
$(-1.25 \pm j 1.3307) / 1.5$. The manipulator systems performance is slower than the previous case with prediction times $T_{1}=0$ and $T_{2}=1$.

\section{Conclusions}

A recently established class of nonlinear predictive continuous-time controllers [1], which have offset removal capability, is generalised to MIMO systems. Elimination of offset or steady-state response error is a major objective in industrial control systems. Formulated within a continuous-time nonlinear generalised predictive control (NGPC) framework, the extension to MIMO systems resolves technical difficulties identified in [1] in a simple and effective manner.

In analogy with the SISO case [1], rather than immediately assuming integral action in the controller, a MIMO nonlinear observer is designed to eliminate offset. The MIMO composite controller consists of an optimal NGPC and a nonlinear disturbance observer to estimate the offset and exhibits a nonlinear PID/PI-like structure. Where this composite controller further differs from [1] even in the SISO case, is that no additional design function for the nonlinear observer is required, and the associated proof of overall nonlinear closed-loop system stability is particularly simple. Moreover, the transient error response of the disturbance observer can be arbitrarily specified by simple design parameters. Very satisfactory performance of the

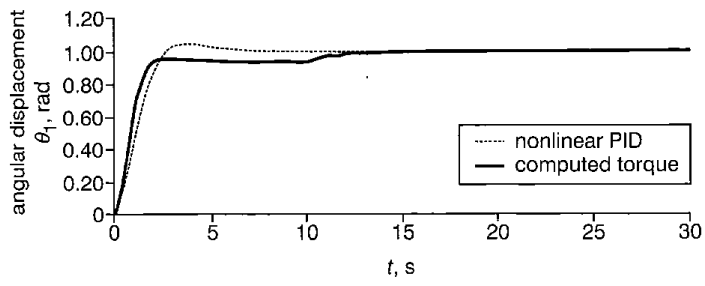

Fig. 4 Link 1, angular displacement step response for predictive times $T_{1}=0, T_{2}=1.5$

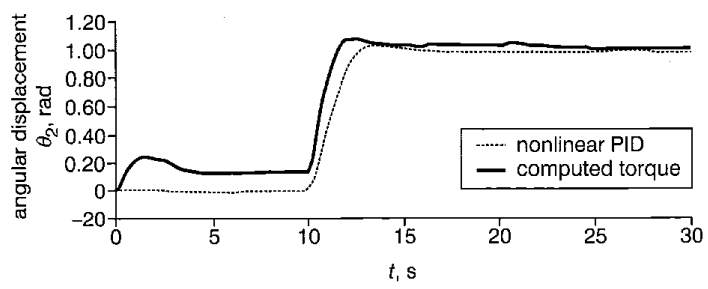

Fig. 5 Link 2, angular displacement step response for predictive times $T_{1}=0, T_{2}=1.5$

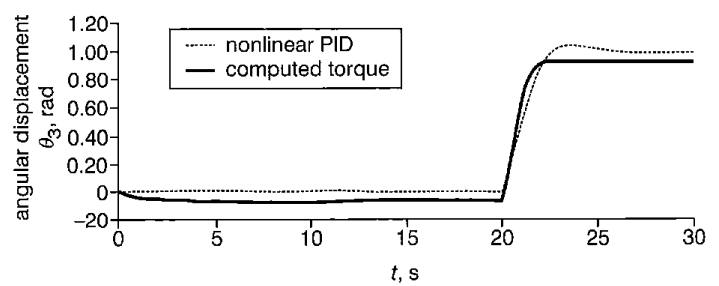

Fig. 6 Link 3, angular displacement step response for predictive times $T_{1}=0, T_{2}=1.5$ 
proposed MIMO nonlinear predictive controller is demonstrated for the independent joint control of a three-link nonlinear robotic manipulator example.

\section{Acknowledgments}

This work was supported by the UK Engineering and Physical Science Research Council under grant ER/L 62665. The authors gratefully acknowledge useful discussions with Professor P.J. Gawthrop on the topic of this paper.

\section{References}

1 CHEN W-H, BALLANCE, D.J, GAWTHROP, P.J., GRIBBLE, J.J, and O'REILLY, J.: 'Nonlinear PID predictive controller', IEE Proc. Control Theory Appl., 1999, 146, (6), pp. 603-611

2 CHEN, W.-H., BALLANCE, D.J., GAWTHROP, P.I., and O'REILLY, J. 'A nonlinear disturbance observer for robotic manipulators', IEEE Trans. Ind. Electron., 2000, 47, (4), pp. 932-937
3 WITTENMARK, B., and ÅSTRÖM, K.J.: 'Simple self-tuning controllers' in UNBEHAUEN, H. (Ed.): 'Methods and applications in adaptive control' (Springer, 1980), pp. 161-198

4 CAMERON, $F$ and SEBORG, D.E.'A self-tuning controller with a PID structure', Int. J. Control, 1983, 38, (3), pp. 401-407

5 LU, P.: 'Approximate nonlinear receding-horizon control laws in closed form, Int. I. Control, 1998, 71, (6), pp. 19-34

6 SOROUSH, M., and KRAVARIS, C.: "A continuous-time formulation of nonlinear model predictive control', Int. $J$. Control, 1996, 63, (1), pp. 121-146

7 GAWTHROP PJ, DEMIRCIOGLU, H, and SILLER-ALCALA, I: 'Multivariable continuous-time generalised predictive control: A statespace approach to linear. and nonlinear systems', IEE Proc., Control Theory Appl., 1998, 145, (3), pp. 241-250

8 CLARKE, D.W., MOHTADI, C., and TUFFS, P.S.: 'Generalised predictive control. Part I: The basic algorithm', Automatica, 1987, 23, (2), pp. $137-148$

9 XIA, X., and ZEITZ, M.: 'On nonlinear continuous observers', Int. J Control, 1997, 66, (6), pp. 943-954

10 CYNCH, KW. SHIROMA, N, ARAI, H., and TANIE, K.: 'Collisionfree trajectory planning for a 3-DoF robot with a passive joint', Int. $J$. Robotics Res., 2000, 19, (12), pp. 1171-1184

11 SLOTINE, J.E., and LI, W.: 'Applied nonlinear control' (Prentice-Hall, Englewood Cliffs, NJ, 1991) 